\title{
Lifelogging Data Validation Model for Internet of Things enabled Personalized Healthcare
}

\author{
Po Yang, Member, IEEE, Danius Stankevicius, Vaidotas Marozas, Member, IEEE, Zhikun Deng, Enjie \\ Liu, Arunas Lukosevicius, Feng Dong, Dali Xu, Fellow, IEEE and Geyong Min, Senior Member, IEEE
}

\begin{abstract}
Internet of Things (IoT) technology offers opportunities to monitor lifelogging data by a variety of assets, like wearable sensors, mobile apps, etc. But due to heterogeneity of connected devices and diverse human life patterns in an IoT environment, lifelogging personal data contains huge uncertainty and are hardly used for healthcare studies. Effective validation of lifelogging personal data for longitudinal health assessment is demanded. In this paper, it takes lifelogging physical activity (LPA) as a target to explore how to improve validity of lifelogging data in an IoT enabled healthcare system. A rule based adaptive lifelogging physical activity validation model, LPAV-IoT, is proposed for eliminating irregular uncertainties and estimating data reliability in IoT healthcare environments. A methodology specifying four layers and three modules in LPAV-IoT is presented for analyzing key factors impacting validity of lifelogging physical activity. A series of validation rules are designed with uncertainty threshold parameters and reliability indicators and evaluated through experimental investigations. Following LPAV-IoT, a case study on a personalized healthcare platform MHA [38] connecting three state-of-the-art wearable devices and mobile apps are carried out. The results reflect that the rules provided by LPAV-IoT enable efficiently filtering at least $75 \%$ of irregular uncertainty and adaptively indicating the reliability of LPA data on certain condition of IoT environments.
\end{abstract}

Index Terms - Internet of things, physical activity, personalised healthcare, data validation.

\section{INTRODUCTION}

$\mathrm{T}$ HE concept of "Internet of Things" (IoT) has become an increasingly growing hot topic within both academia and industry [1-6]. The fundamental idea of IoT is to build up a

This work was supported in part by CARRE (No. 611140) and MHA (No. 600929) projects, funded by the European Commission FP 7 programme.

P. Yang (corresponding author) is with the School of Computer Science, Liverpool John Moores University, Liverpool, L3 3AF, UK. (e-mail: p.yang@ljmu.ac.uk).

Z. Deng, E. Liu, and F. Dong are with Centre of Computer Graphics and Visulisation, Bedfordshire University, Luton, LU1 3JU, UK. (e-mail: zhikun.deng@beds.ac.uk, enjie.liu@beds.ac.uk, feng.dong@beds.ac.uk).

V. Marozas, D. Stankevicius, A. Lukosevicius is with Department of Electronics, Kaunas University, Kaunas, Lithuania. (e-mail: varimao@ktu.lt, dainius.stankevicius@ktu.lt, arunas.lukosevicius@ktu.lt).

L. D. Xu is with the Old Dominion University, Norfolk, VA 23529, USA, and also with the Institute of Computing Technology, Chinese Academy of Sciences, Beijing 100190, China (e-mail: LXu@odu.edu).

G. Min is with the Department of Mathematics and Computer Science, Exeter University, Exeter, EX4 4QF, UK (e-mail: g.min@exeter.ac.uk). globally interconnected continuum of a variety of objects in the physical environment. With the pervasive utilization of heterogeneous sensors - such as accelerometers, gyroscopes, altimeters, temperature, pressure, humidity, UV radiation, Radio-Frequency Identification (RFID) tags and other portable low-cost devices, significant advancements in the IoT have generated a large amount of opportunities in industrial areas [6-11], particularly in healthcare field [12-18].

Due to the exponential growth of commercial wearable devices and mobile apps [22-26], it has become increasingly possible to remotely monitor a patient or citizen's health by connecting heterogeneous medical devices into an IoT platform [18-20]. A promising trend in healthcare fields appears that the IoT enabled technology is transforming traditional hubs of healthcare to personalized healthcare systems and especially mobile environments. However, using IoT enabled technology in healthcare systems is challenging considering heterogeneity of connected wearable devices, high volume of generated multi-dimensional personal health data, and privacy issues. These issues lead to huge uncertainty in lifelogging personal health data. Effective validation of these high volume and multi-dimensional health data becomes a major demand on IoT personalized healthcare systems.

Technically and functionally sophisticated wearable devices and mobile applications [29-31] enable recording a variety of lifelogging personal health information; including physical activity, weight, sleep quality, heart rate, blood pressure, etc. Among this data, physical activity is mostly well-observed due to the maturity of microelectromechanical systems (MEMS) based accelerometer technology as well as easily and openly accessible Global Position System (GPS). Numerous research works [21-28] and commercial products [29-31][33] have attempted to accurately monitor physical activity and access activity patterns and intensity level, by using either dedicated wearable sensors [29-31] or advanced machine learning algorithms [22-25]. But these studies mostly depend on performance optimization of single sensor or a combination of GPS and accelerometer by analyzing raw sensors' signals. In IoT personalized healthcare environments, physical activity data is discretely daily basis from globally heterogeneous third party devices. Traditional physical activity validation methods hardly deal with these scattered and heterogeneous data. Also, due to diversity and changes of personal lifestyles, lifelogging physical activity data in IoT enabled personalised healthcare systems has remarkable uncertainties. Effective validation of 
these data from heterogeneous devices is an essential but highly difficult task. The requirements of customization and longitudinal study in an IoT healthcare environment make this task ever harder. Our study in this paper attempts to take lifelogging physical activity (LPA) as a target to explore the how to improve validity of lifelogging data in an IoT based healthcare environment.

This paper investigates the problem of effectively validating lifelogging physical activity in a heterogeneous devices based IoT enabled personalized healthcare environment. A rule based adaptive lifelogging physical activity validation model, LPAV-IoT, is proposed for eliminating irregular uncertainties and estimating physical activity data reliability in IoT enabled personalized healthcare systems. It enables data validation procedure in IoT environments to be a dynamic standardized empirical analysis workflow with four layers including factors, methodologies, knowledge and actions. The factors impacting the validity of physical activity are categorized into device, personal and geographic. Each factor defines a longitudinal data analysis based investigation strategy. The validation rules are represented with a set of uncertainty threshold parameters and reliability indicators, which can be initiated by historical data and adaptively updated regarding the needs of an IoT enabled personalized healthcare system. The effectiveness of LPAV-IoT is verified by carrying out a case study on an IoT enabled healthcare platform MHA [38] with state-of-the-art wearable devices and mobile apps are carried out. The results reflect that the validation rules and action criteria delivered by LPAV-IoT effectively improve the validity of lifelogging physical activity data in the MHA system. LPAV-IoT provides an efficient and adaptive solution for the validation of IoT environment based lifelogging physical activity data. The main contributions are below:

1. A rule based lifelogging physical activity validation model, LPAV-IoT, is proposed for effectively eliminating irregular uncertainties and estimating physical activity data reliability in IoT enabled personalized healthcare systems.

2. A series of validation rules representing with uncertainty threshold parameters and reliability indicators are designed and evaluated through a set of experimental investigation. These rules are capable of being adaptively and dynamically updated regarding the needs of an IoT enabled personalized healthcare system.

3. A case study on an IoT enabled healthcare platform MHA [38] with heterogeneous devices is provided to evaluate the proposed validation rules and action criteria. A discussion and analysis on experimental results are given.

The rest of the paper is structured as follows. Section II reviews related work. Section III presents the description of LPAV-IoT model. Section IV gives experimental investigation with LPAV-IoT model. Section V reports a case study that applies LPAV-IoT model in MHA platform [38]. Section VI and VII provide the discussions and conclusions.

\section{RELATED WORK}

IoT based personalized healthcare systems [14] use a set of interconnected devices to create an IoT network devoted to healthcare assessment. It will observe and collect personalised health information from different wearable sensing devices through a middleware that provides interoperability and security needed in the context of IoT for healthcare. These wearable devices are capable of recording multiple type health data, including physical activity, weight, sleep, heart rate and blood pressure. Among these data, physical activity is mostly well-observed.

As a major risk measure for chronic diseases, daily physical activity recognition and monitoring with wearable sensors have been investigated by a number of researchers [21-28] [29-34]. In [22-23], authors carry out a study on recognizing and classifying physical activity by analyzing signal features from 3D (triaxial) accelerometers on hip and wrist and GPS data with a hybrid classifier of custom decision tree and neural networks. The results are reported a classification accuracy up to $89 \%$ for detecting 10 daily actions. ProeTex [24] project develops an algorithm that combines features of ECG and triaxial accelerometer in smart garments for detecting nice classes of physical activity with overall classification accuracy up to $88.8 \%$. In [27-28], researchers have integrated on-body sensors in a wireless network for the purpose of activity recognition and lifestyle monitoring. Authors in [27] utilize a network of five accelerometers to classify a sequence of 20 daily activities with accuracy of $84 \%$. The system in [28] that uses seven different sensors embedded in a single node, including microphone, phototransistor, 3D accelerometer, 2D compass, barometer, ambient light and digital humidity, to classify 12 movements with accuracy up to $90 \%$. All aforementioned work on daily physical activity recognition has achieved high classification accuracy of recognizing multiple daily activity actions. But all of these studies rely on a collection of physical activity data as a raw accelerometers' signals. In IoT healthcare systems, LPA mostly data comes from a variety of third party mobile devices. Traditional PA classification methods [21-28] are infeasible to handle these scattered and heterogeneous physical activity data.

Recently, many commercial wearable products [29-30] and mobile applications [31-32] [36] have been released for the long term record and collection of personal LPA. The most famous mobile apps, such as Moves, are based on smartphone 3D accelerometer data and GPS information which allows tracking user movement activities including location, distance and speed. The wearable products, such as Fitbit Flex, Nike+ Fuelband, Withings, are all wristband devices that record steps count, distance, and calories burnt. These wearable devices communicate with mobile phone via Bluetooth employing relevant mobile applications. While above products have been proven its popularity among general users, their major usages are limited in the fitness fields. It is due to diversity of life pattern and environmental impacts; personal LPA data from individual wearable device exhibits remarkable uncertainty. The validating of these physical activity data in longitudinal healthcare cases is extremely challenging. Also, as the exponential growth of mobile healthcare market, numerous similar wearable products have been developed, which will significantly increase the heterogeneity and diversity of devices connected in IoT based personalized healthcare systems. Effective validation of physical activity data from heterogeneous devices in IoT enabled personalized healthcare environments becomes more difficult. 


\section{LPAV-IOT MODEL}

\section{A. LPAV-IoT Ecosystem}

The ecosystem for LPAV-IoT is the theoretical cornerstone of validating of physical activity in an IoT environment, as shown in Fig.1. In terms of the concept of IoT, personal health data are accumulated and measured as a cube in three dimensions (3D): Persons, Devices and TimeLine. The increment in any dimension results in an expansion of the health data grid. The products like Fitbit or Moves [29] occur on a 2D plane (Persons $\times$ TimeLine), which refer to scenarios that single device is used by increasing population over time. Physical activity recognition with sensor fusion [21-28] appears on a 2D plane (Devices $\times$ TimeLine) for classifying individual person's activities with historical health data. The target of LPAV-IoT model is to deal with a 3D cube of rapid-growth lifelogging physical activity (LPA).

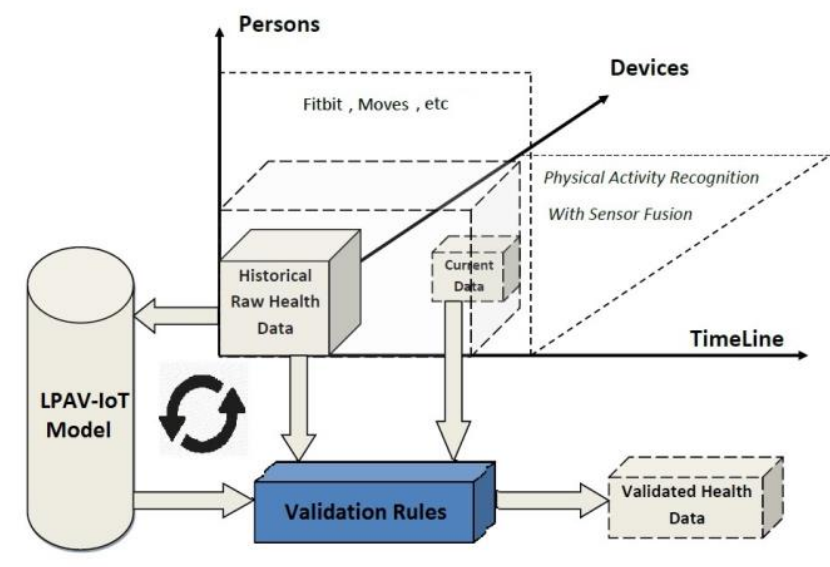

Fig. 1 Concept of IoT personalized healthcare systems

The workflow of LPAV-IoT model for validating physical activity is a dynamic recurrence by duration along the timeline. The validation rules are initiated by feeding a set of historical raw physical activity data in the LPAV-IoT model; then are used to validate the current physical activity. After a period, historical raw physical activity data is expanded with more users or devices over time. The validation rules are dynamically changed and updated by feeding new historical physical activity data into the LPAV-IoT model. Also, LPAV-IoT model provides a configuration to register the information on person and devices dimensions. It adaptively supports the need from different users or groups. The concept of LPAV-IoT model is to firstly identify the key influencing factors with detailed issues causing uncertainty of lifelogging physical activity; and design a series of benchmarks and experimental study methods for qualitatively evaluating these influencing factors. Through these experiments, LPAV-IoT model enables delivering a practically efficient validation strategy containing a series of validation principles, rules and actions. Fig. 1 shows a conceptual diagram of LPAV-IoT model with three aims:

Uncertainty Reduction: To offer approaches for effectively filtering errors and reducing uncertainty of LPA data.

Reliability Estimation: To provide indicators for estimating the reliability of LPA data on certain conditions of IoT system.
Adaptivity: To generally support a variety of heterogeneous mobile devices. The validation rules of this model have to be adapted to fit to the IoT healthcare application situations.

Also, LPAV-IoT is desirably extendible and scalable for supporting emerging technological possibilities of devices in an IoT healthcare environment. New unidentified influencing factors can be added in the LPAV-IoT model and investigated with a similar evaluation methodology.

\section{B. Uncertainty Classification}

In IoT healthcare environments, uncertainties of LPA here can be categorized into two types:

Irregular uncertainty: Irregular Uncertainty (IU) occurs randomly and accidently in lifelogging physical activity data. The causes of these uncertainties include device malfunctions or faults, breakdown of third party server, misuse of mobile apps, sudden change of personal circumstance. The occurrence of irregular uncertainty will appreciably impact the efficiency and accuracy of assessing personal health.

Regular uncertainty: Regular Uncertainty (RU) occurs frequently and persistently in lifelogging physical activity data. The causes resulting in these uncertainties are mainly from some regular influencing issues, like intrinsic sensors' errors, differentiation of personal physical fitness and changes of environment. The occurrence of regular uncertainty in physical activity data is inevitable so that it is impossible to completely eliminate these uncertainties.

LPAV-IoT aims at delivering methods for eliminating the impact of irregular uncertainty and managing the impact of regular uncertainty.

\section{Impacting Factors Analysis and Matrix}

While irregular uncertainties occur accidently and are hardly quantified by impacting factors, their occurrence frequency is relatively low over time. A statistical analysis in historical data can detect threshold parameters to filter them. Daily physical activity is mainly measured as daily steps $\left(S_{d}\right)$, daily walking distance $\left(D_{d w}\right)$ and daily average walking speed $\left(V_{\text {daw }}\right)$ as it is shown in Table I. It is believed that the majority of daily steps and daily average walking speed have to be in a specific range. Two threshold parameters $\left(\mathrm{T}_{\mathrm{s}}\right.$ and $\left.\mathrm{T}_{\mathrm{v}}\right)$ are defined to filter the irregular uncertainties regarding a probabilistic distribution.

For regular uncertainties, the impacting factors in LPAV-IoT are categorized into three modules, which are device factors, personal factors and geographic factors. In the device factors module, existing popular wearable devices or mobile apps are classified by sensory technique into three types: GPS based, Accelerometer based, a combination of sensors based. The accuracy of these three sensory techniques for measuring step count and distance are quantified by Mean of relative error and Standard Deviation of relative error though a series of experiments.

The personal factors module studies if the differences of human demographic, anthropometric and fitness data give regular uncertainties to LPA data. These differences usually include the age, gender, height, weight and medical history, etc. The information relies on users' efforts of manual input, which maybe incomplete. There is a need for a benchmark 


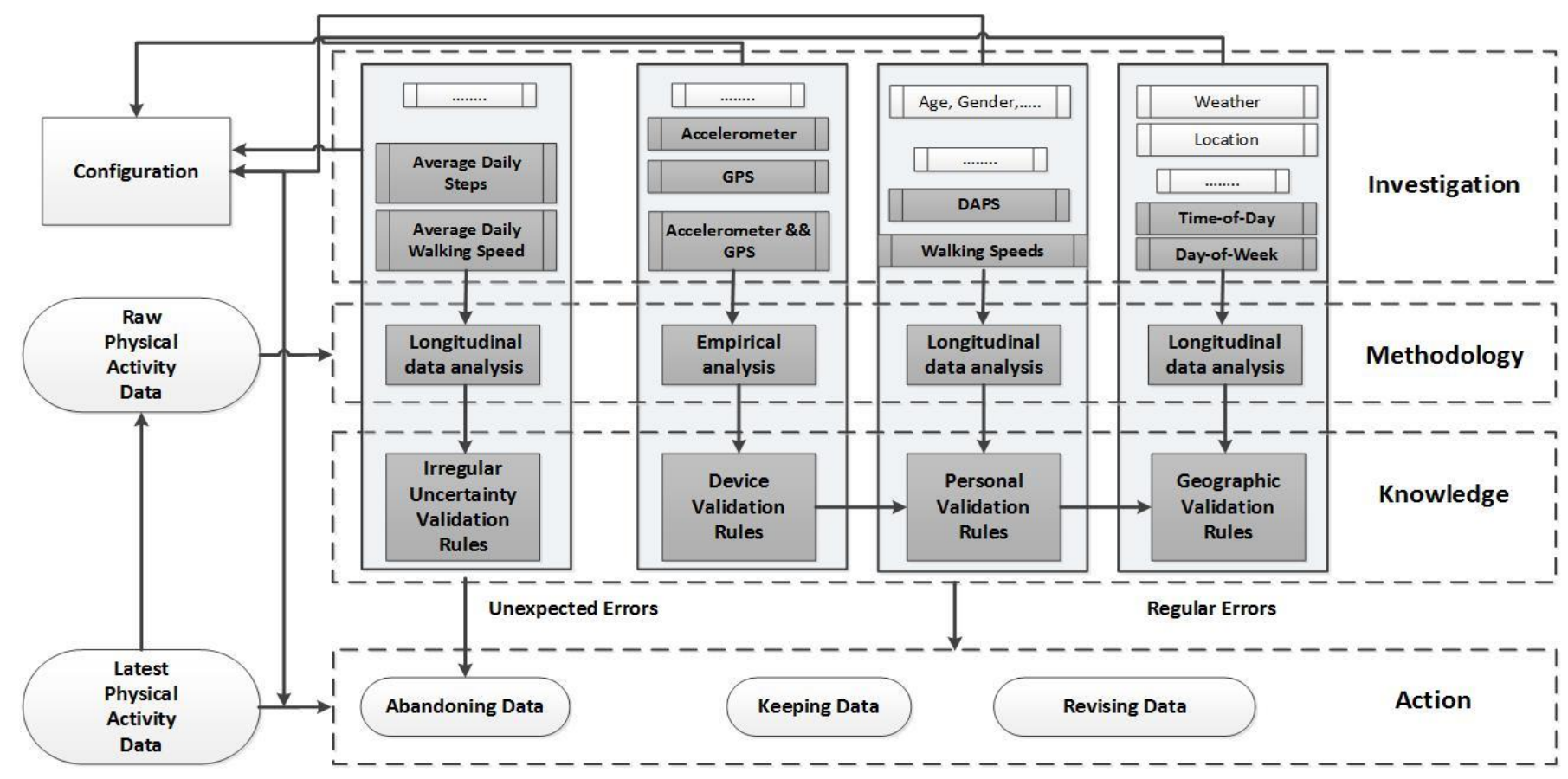

Fig. 2 Diagram of LPAV-IoT Model

to represent a person's physical fitness from completed data sources. Here a walking speed related score is defined to represent a person's physical fitness, named as Daily Activity in Physical Space (DAPS). This score is inspired from work [34] that proposes a Movement and Activity in Physical Space (MAPS) score as a functional outcome measurement for encompassing both physical activity and environmental interaction. Currently, most of wearable devices or mobile apps have provided the third party APIs to assess the intensity of physical activity regarding walking speed. For instance, Fitbit classifies the intensity of daily activities into Very Active, Moderately Active, Lightly Active and Sedentary; Moves records a series of walking segments containing duration, distance and speed. Here, we classify the intensity of daily physical activity into $N$ levels in terms of the ranges of walking speeds $\left(\mathrm{V}_{1}, \mathrm{~V}_{2} \ldots \mathrm{V}_{\mathrm{n}}\right)$. The DAPS formula is created by summing these different level walking speeds:

$$
D A P S=\sum_{1}^{N} V_{t}
$$

For understanding the impact of personal factors on measure of daily physical activity, we give two hypothesis tests that:

1) Person's physical fitness has a strong relationship with his daily physical activity. A person with strong physical fitness shall have a high value of daily physical activity.

2) For a group of population having similar lifestyle, regular uncertainties raised by personal factors are supposed to follow a linear relationship with Daily Steps. A person walks more steps or distances, regular errors will be increased linearly in daily steps. A proportional function (2) is defined for representing their relationship, where $\beta$ is a proportion ratio.

$$
\operatorname{Err}_{p}=\beta \times S_{d}
$$

In order to testing our hypothesis, Pearson's correlation coefficient (r) is simply used in a group of persons to measure the strength of the association between $\operatorname{Err}_{\mathrm{p}}$ and daily physical activity $\left(\mathrm{S}_{\mathrm{d}}\right.$ or $\left.\mathrm{D}_{\mathrm{dw}}\right)$. If personal factor (physical fitness) has a strong impact on regular uncertainties, all persons' Pearson figure $r$ will be close to 1 or -1 . It is noteworthy that the motivation of LPAV-IoT model aims at providing an investigation approach for improving the validity of generic lifelogging physical activity in an IoT environment. It does not only aim at dealing with the intensity of physical activities (IPA), and can be extended to apply into more complex physical activity related subjects. But most available mobile apps or wearable devices only release API to access limited type of physical activity data, which is the intensity of physical activity. Thus, LPAV-IoT model aims at building up a set of investigation methods for some data, which are able to be collected and evaluated by experiments. IPA being improved by LPAV-IoT model may be not remarkable to the index based representation for users.

The geographic factors module aims at investigating the impacts of location specific information related contextual data on the accuracy of daily physical activity. This information can include Time (time of day, life events, i.e.), Location (country, part of city, "at work" etc.), Environmental factors (weather conditions, etc.). Considering the difficulty of establishing and recording completed user life and environment profiles, we only list three items in geographic factors: weather, hourly-change of physical activity, and weekly-change of physical activity. The changes of daily physical activity over these three issues are measured with statistical analysis in historical data. A few range and type of parameters are defined in Table 1. A reliability indicator $(\mathrm{R})$ for estimating the overall impact of above three impacting factors is formulated below: 
TABLE I

LISTING OF PARAMETERS AND INDICATORS IN LPAV-IOT MODEL

\begin{tabular}{|c|c|c|c|}
\hline & & Parameters & Descriptions \\
\hline \multirow{3}{*}{\multicolumn{2}{|c|}{ Raw Physical Activity Data }} & $\mathrm{S}_{\mathrm{d}}$ & Daily walking steps \\
\hline & & $\mathrm{V}_{\text {daw }}$ & Average daily walking speed \\
\hline & & $\mathrm{C}$ & Confidence interval for filtering historical data distribution \\
\hline \multicolumn{2}{|c|}{ Irregular Uncertainty } & $\mathrm{T}_{\mathrm{v}}$ & Threshold parameter for filtering incorrect average daily walking speed \\
\hline \multirow{8}{*}{$\begin{array}{l}\text { Regular } \\
\text { Uncertainty }\end{array}$} & \multirow[b]{2}{*}{ Devices } & ES_mean & Mean of step count relative error \\
\hline & & ES_std & Standard Deviation of step account relative error \\
\hline & \multirow[t]{2}{*}{ Personal } & DAPS & Daily Activity in Physical Space score \\
\hline & & $\mathrm{V}_{1}, \mathrm{~V}_{2} \ldots \mathrm{V}_{\mathrm{n}}$ & Average Walking Speed regarding intensities of daily physical activity. \\
\hline & \multirow{4}{*}{ Geographic } & Sh(morning, afternoon, night) & Daily steps range in morning, afternoon and night \\
\hline & & Dh( morning, afternoon, night ) & Daily walking distance range in morning, afternoon and night \\
\hline & & Swk(working, weekend) & Daily steps range in working days and weekend \\
\hline & & Dwk(working, weekend) & Daily walking distance range in working days and weekend \\
\hline
\end{tabular}

$$
R=D \times P \times E
$$

Where:

D: Reliability of device factors on physical activity

P: Reliability of personal factors on physical activity

E: Reliability of geographic factors on physical activity

\section{Data Validation Strategy}

Data validation strategy aims at conducting a set of validation rules for eliminating irregular uncertainties and reducing the impacts of regular uncertainties on LPA data. This strategy is designed by using a combination of statistical analysis methods on longitudinal studies and experimental analysis approaches. The workflow of data validation strategy is presented as 4-layers structure in Fig.2.

Investigation Level: to provide analysis and classification of detailed influencing items in each impacting factor module, also establishes corresponding uncertainty measurement matrix. A notable feature of influencing items level is extendibility which means that it may add more items into the LPAV-IoT for further investigation.

Methodology Level: to designs investigation approaches for each impacting factor module regarding identified items and established matrix. They include statistical longitudinal data analysis and experimental based empirical analysis methods.

Knowledge Level: to conduct validation rules and principles following the investigation approaches. These rules aim at quantitative removal of irregular uncertainties, and qualitative exploration of the relationship between impacting factors and regular uncertainty.

Action Level: to contain options of executed actions on physical activity data regarding validation rules. Three main types of actions are given in the model: to abandon data, to keep data and to revise data. The purpose of LPAV-IoT is to validate and verify physical activity data, so the action of revising data is not considered in this paper.

The steps of data validation strategy are described below:
For removing irregular uncertainty:

1. To configure the information related to impacting factors and collect certain type of raw historical PA data.

2. To calculate the parameters $S_{d}, D_{d w}, V_{\text {daw }}$ with raw data.

3. To plot the data of $S_{d}, D_{d w}, V_{\text {daw }}$ in line and calculate the value of $T_{s}$ and $T_{y}$ with eclipse filtering equation to cover data with a confidence interval of $95 \%$.

4. To use $\mathrm{T}_{\mathrm{s}}$ and $\mathrm{T}_{\mathrm{y}}$ for removal of irregular uncertainty PA data. 5. To circulate above process in another period with updated raw data.

The rules are: a) Following eclipse filtering equation, we can get the value of $T_{s}$ and $T_{y}$. b) For a daily PA data, if daily walking steps is lower than $T_{s}$, or average daily walking speed is lower than $\mathrm{T}_{\mathrm{y}}$, we will abandon this data.

For device factors:

1.To list and classify typical wearable devices and mobile applications for PA data recording.

2. To design a set of evaluation experiments including daily activities, like walking for measuring accuracy parameters of the devices: Es_mean, Es_std, Ed_mean and Ed_std (see Table 1 for definitions).

3. To conduct the experimental findings as validation rules and establish the equation for device reliability indicator $\mathrm{D}$.

4. To circulate the above process with new types of devices.

The rules are: a) Following designed experiments including $N$ subjects; we can get the device reliability indicator $D_{n}$ for each subject. b) The overall reliability of the device is formulated as a combination of these separate reliability indicators (4):

$$
D=\sum_{n} k D_{n}
$$

Where:

$D$ : overall reliability of the device for physical activity;

$D_{n}$ : reliability of one subject;

$k$ : weight of each parameter reliability. 
For personal factors:

1. To calculate the value of $\mathrm{V}_{1}, \mathrm{~V}_{2} \ldots \mathrm{V}_{\mathrm{N}}$ with raw historical physical activity data by individual person.

2. To calculate the value of DAPS by summing up $V_{1}, V_{2} \ldots V_{N}$. 3. To calculate the value of Pearson Correlation $r$ between DAPS and $S_{d}$ or $D_{d w}$ by individual person.

4. To conduct the experimental findings as validation rules and establish the formula for personal reliability indicator $\mathrm{P}$.

5. To circulate the above process with more subjects.

The rules are: a) On the condition that we get every individual's Pearson Correlation. b) If the Pearson Correlation $r$ from individuals is diverse, it means that no strong impact of daily speed or MAPS on daily steps. Personal factors (for normal people) will not generate significant errors in physical activity data. c) If the Pearson Correlation $r$ from individuals is nearly identical, it means that Personal Factors (for normal people) will generate significant errors in physical activity data. d) The reliability of estimating personal factors on PA is measured by the difference of individual person's DAPS and a standard DAPS in a group $M$ of populations. If it is assumed that $M$ subjects' DAPS data is recorded in the platform, the reliability of estimating personal factors on physical activity is formulated below:

$$
\begin{gathered}
\overline{D A P S}=\frac{\sum_{m=1}^{M} D A P S_{m}}{M} \\
P=1-\sqrt{\left(\frac{D A P S_{i}-\overline{D A P S}}{D A P S_{i}}\right)^{2}}
\end{gathered}
$$

Where:

$P$ : overall reliability of personal factors for physical activity;

$D A P S$ : Daily Activity in Physical Space Score

$M$ : Total number of persons in the group.

For geographic factors:

1. To classify and categorize physical activity data regarding weather, hourly-change and weekly-change parameters.

2. To plot the data of $S_{d}, D_{d w}, V_{d a w}$ in line and calculate the range value of parameters to cover a confidence interval of $95 \%$.

3. To conduct the experimental findings as validation rules and establish the formula for personal reliability indicator $\mathrm{E}$.

4. To circulate the above process in another period with updated raw data.

The rules are: a) The reliability of estimating geographic factors on physical activity can be measured by the difference between individual daily steps and average daily steps in weekdays by devices. If it is assumed that $M$ person wears one type device, his / her steps data in weekdays are recorded as $\mathrm{Swk}^{\mathrm{t}}(\mathrm{t}=1, . ., 7)$, the reliability of estimating geographic factors on physical activity is formulated below:

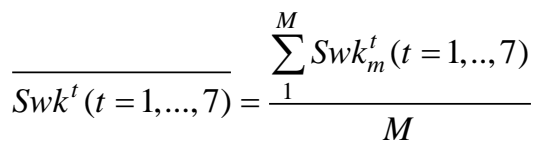

$$
E=1-\sqrt{\left(\frac{S w k_{i}^{t}(t=1, \ldots, 7)-\overline{S w k^{t}(t=1, \ldots, 7)}}{S w k_{i}^{t}(t=1, \ldots, 7)}\right)^{2}}
$$

Where:

$\mathrm{t}$ : represents weekdays from Monday to Sunday.

Swk : walking steps on certain day in a week.

\section{E. Adaptability and Extendibility}

The design of LPAV-IoT model aims at generic utilization in IoT enabled personal healthcare systems. Configuration is defined here in the LPAV-IoT model for registering the information regarding devices factor, personal factor or geographic factor. By using this information, LPAV-IoT model is capable of adaptively adjusting the values of parameters in validation rules to account for different needs. LPAV-IoT model is able to adapt itself efficiently; it is fast in responding to changed settings or needs in an IoT enabled healthcare environment. Also, more extended feature of LPA-IoT model are discussed in section VI.

\section{EXPERIMENTAL INVESTIGATION}

In theory, LPAV-IoT model aims at validating lifelogging physical activity in an IoT healthcare environment with any population, for any devices and at any time periods. This paper takes two EU healthcare projects: MHA [38] and CARRE [37] as case studies to verify the effectiveness of LPAV-IoT model. This section presents the establishment of validation rules with LPAV-IoT by MHA and CARRE projects. The evaluation of device factors modules include 7 typical physical activity recorders used in CARRE project: Fitbit Flex, Fitbit One, iHealth AM3, Medisana Vifit Connect, Withings Pulse O2, Jawbone UP24 and Moves. The evaluation and validation of irregular uncertainty, personal and geographic factors are based on MHA platform, which is an IoT enabled personal healthcare experiment platform connecting Moves, Fitbit and Withings. This platform enables user to transfer their physical activity data from these third party providers into MHA server, and then to be able to visualize and analyse this information for a better user understanding and experiences.

\section{A. Irregular Uncertainty}

Eliminating irregular uncertainties is the primary step of data validation strategy in LPAV-IoT model. On MHA platform, we initially collect daily physical activity (Steps, Distance and Calories) of 7 users over 6 months by 3 types of wearable devices of recorders (Withings, One and Moves). All these 7 users ( 1 female and 6 male) are researchers in university, and their ages are in the range of 30-50 years old. The features of this raw activity data are: 1) All 7 people use Moves, 2 of them additionally use Withings, and another 3 people use Flex. 2) Missing data occurs frequently in Withings and Flex, because users easily forget wearing them. 3) Some data in Flex shows lower steps, which is probably because users take off their wearable devices some time, or devices are out of battery. 4) Moves data are more completed than Flex or Withings, but with relatively high errors. Following data validation strategy in section V.D, we calculate $\mathrm{V}_{\text {daw }}$, and plot $S_{d}$ and $V_{\text {daw }}$ in $2 D$ diagram as in Fig.3. 


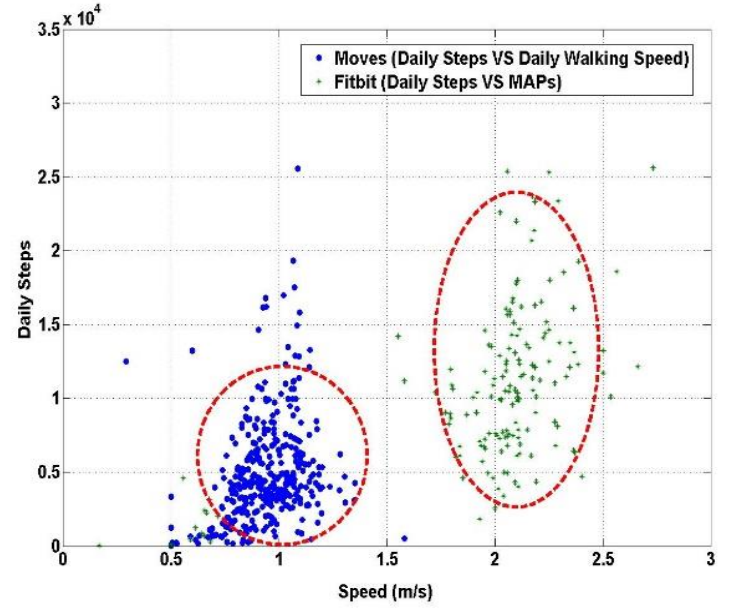

Fig. 3 Distribution of Irregular Uncertainty

Fig.3 demonstrates that: daily steps of individual by Moves are about 4000 - 7000; Flex or Withings give daily steps about 6000 - 13000; Moves gave a lower measurement of daily steps than Flex or Withings on the same condition. Normal people should have a daily steps in the range 1000-20000. Flex and Withings sometimes show daily steps below 1000 .

In order to measure $\mathrm{T}_{\mathrm{s}}$ and $\mathrm{T}_{\mathrm{y}}$ to remove irregular uncertainty physical activity data [30], we use an eclipse equation (9) to cover $95 \%$ of data $(C=0.95)$.

$$
\frac{(x-h)^{2}}{a^{2}}+\frac{(y-k)^{2}}{b^{2}}=1
$$

Where:

$h$ : Average daily walking speed

$k$ : Average daily walking steps

$a$ : Error range of average daily walking speed

$b:$ Error range of average daily walking steps

A noticeable issue here is that we only consider the lower limits of walking steps and the upper limits of walking speeds as threshold parameters. On some days users might walk distinctly more steps than usually, while the other days might be more sedentary. The threshold parameters are represented in equation (10):

$$
\begin{aligned}
& T_{y}=h+a \\
& T_{s}=k-b
\end{aligned}
$$

The rules are: a) Following equation 4, we can get $\mathrm{T}_{\mathrm{s}}=68$, and $\mathrm{T}_{\mathrm{y}}=0.56$ for Moves, and $\mathrm{T}_{\mathrm{s}}=1329$, and $\mathrm{T}_{\mathrm{y}}=1.67$ for Flex. $\mathrm{b}$ ) For a daily physical activity data recorded by Moves, if daily walking steps is lower than 68, or average daily walking speed is lower than 0.56, we will abandon this data. c) For a daily physical activity data recorded by Fitbit, if daily walking steps is lower than 1329, or average daily walking speed is lower than 1.67, we will abandon this data.

\section{B. Device Factor}

The characteristic evaluation of device factors in LPAV-IoT model presents design and results of experimental investigation that carried out in order to evaluate the accuracy of wearable equipment. A total of 6 devices were included in this study: Flex, One, iHealth, Vifit, Withings, Jawbone. All these devices are classified as an "accelerometer only" based physical activity trackers. They were chosen from the market as the suitable devices for long term physical activity monitoring due to low price, long battery life, compatibility with Android and the most importantly - API availability. The Moves app was included in the evaluation as it is the only piece of equipment employing both GPS and accelerometer technology with available API. Two more apps were included in the study as the "GPS only" equipment: Endomondo and Google MyTracks. The same main criterion - API availability- was applied when choosing the GPS enabled apps.

The study was performed in two stages: the primary and final investigations. In both parts, some of the physical activity parameters available from the selected devices were measured on healthy volunteers and compared to the reference parameters. Some of the devices are suitable to wear on the wrist, others - on the waist or in the pocket and some provide the ability to choose how to wear them. The wrist wearing site was preferred during the experimentation since it allows easy and unobtrusive non-stop physical activity tracking. In the primary investigation, three variables were measured - steps taken, distance travelled and calories burned. All accelerometer based devices output these three parameters, while Moves outputs only the step count and the distance and the "GPS only" apps output only the distance and the calories. The reference method for measuring the step count consisted of raw accelerometer signals acquired by the custom physiological and kinematical signal recorder KTU BMII Cardiologer v6 attached to the waist, and a semi-automatic peak detection algorithm implemented in Matlab. The reference method for measuring calories was indirect calorimetry implemented in portable calorimeter Cosmed K4b2. Since this calorimeter is enabled with a GPS module, it also was used as a reference method for measuring travelled distance. 4 healthy volunteers participated in this part. The experimentation protocol was below for each participant:

1. A short walk within fixed distance of $160 \mathrm{~m}$ (80 meters back and forth with stopping) where only the step count was measured. "GPS only" devices were not included.

2. Calculation of the average step length using the distance and the step count from the reference method.

3. Update of the devices with personal information, such as birth date, height, weight, step length, running step length.

4. The approximate of 1000 meters long casual walking exercise via fixed rounded route. The participant was able to choose his/her own walking pace.

5. Jogging exercise of $200 \mathrm{~m}$ (100 m back and forth without stopping). "GPS only" devices not included.

6. Slow walking exercise of 200 meters (100 m back and forth without stopping). "GPS only" devices not included.

7. Stair climbing exercise (5 floors). "GPS only" devices not included.

The protocol includes two parts. One part includes the most frequent physical activity - walking (exercises 1, 4 and 6). The other part includes less frequent physical activity (exercises 5 and 7). The results from this primary evaluation are also divided into two parts respectively. The error ranges for each 
type of devices are presented in Table II and Table III. While Moves app output the distance information, it was not accurately recorded by the operator. So this data was discarded from the investigation. GPS devices data was not acquired during the less frequent exercises. These results present only the preliminary error ranges of the devices, but they create some guidance for further experimentation. The calories estimation from the accelerometer devices shows the worst performance, while accelerometer plus GPS devices do not output such information at all. Cosmed K4b2 calorimeter is also very complicated for the participants to work with. So the calories estimation comparison was excluded from the further experimentation. On the other hand, the GPS devices showed very good performance in measuring distance. It was decided to replace the reference GPS device with the GPS enabled app in the smartphone (Tracks). In order to simplify the exercises in the experimentation and due to some limitations (GPS not working inside the building), it was decided to exclude the less frequent exercises from the experimentation. The reference method for counting steps remained the same as in the primary investigation. 6 healthy volunteers participated in the second investigation. A new experimentation protocol was below:

1. A short walk within fixed distance of $100 \mathrm{~m}$ (50 meters back and forth with stopping) where only the step count was measured.

2. Calculation of the average step length using the distance and the step count from the reference method.

3. Update of the devices with personal information, such as birth date, height, weight, step length, running step length.

4. An approximate of 1000 meters long casual walking exercise via fixed rounded route. The participants can choose their own walking pace. Step count and distance was measured.

The first short experiment shows the ability of the devices to accurately capture short episodes of physical activity (e.g. walking in the office). The long walk experiment shows the ability to accurately record the most frequent daily physical activity - casual walking (e.g. walking to/from work). The results as a mean of error and the STD of error are presented in Table IV for each device and each measured variable separately. These results show that devices based on the same accelerometer technology perform differently and could not be used interchangeably. It may seem that the wrist wearing site can cause problems as the Flex tracker has lower accuracy than One. On the other hand, we can see that Withings performs similarly to the One while also worn on the wrist. The error ranges were updated according to the results of the final investigation and are presented in Table $\mathrm{V}$. The actual ranges are lower than in primary investigation. Another observation is that Accelerometer + GPS devices have slightly lower error range for step count and significantly lower error range for distance estimation. So we propose that the device reliability factor should be separately calculated for each of the measured parameters. In this particular case with two parameters, the following two equations are introduced:
TABLE II

THE ERROR RANGES FOR WALKING EXERCISES

\begin{tabular}{|c|c|c|c|}
\hline & \multicolumn{3}{|c|}{ Error range (min - max), \% } \\
\hline Device type & Steps & Distance & Calories \\
\hline Accelerometer & $0,0-82,5$ & $0,1-68,1$ & $0,2-93,3$ \\
\hline Accelerometer + GPS & $4-56,4$ & N/A & N/A \\
\hline GPS & N/A & $0-5,4$ & $2,4-45,8$ \\
\hline \multicolumn{4}{|c}{ TABLE III }
\end{tabular}

THE ERROR RANGES FOR LESS FREQUENT EXERCISES

\begin{tabular}{|c|c|c|c|}
\hline & \multicolumn{3}{|c|}{ Error range (min - max), \% } \\
\hline Device type & Steps & Distance & Calories \\
\hline Accelerometer & $0,0-74,6$ & $0,7-72,4$ & $6,4-80,6$ \\
\hline Accelerometer + GPS & $6,9-94,2$ & N/A & N/A \\
\hline GPS & N/A & N/A & N/A \\
\hline \multicolumn{4}{|c}{ TABLE IV }
\end{tabular}

THE ACCURACY OF THE DEVICES

\begin{tabular}{|c|c|c|c|c|c|c|}
\hline \multirow{2}{*}{ Device } & \multicolumn{2}{|c|}{$\begin{array}{c}\text { Error in steps } \\
\mathbf{1 0 0} \mathbf{~ m}\end{array}$} & \multicolumn{2}{c|}{$\begin{array}{c}\text { Error in steps } \\
\mathbf{1 0 0 0} \mathbf{~ m}\end{array}$} & $\begin{array}{c}\text { Error in } \\
\text { distance } \\
\mathbf{1 0 0 0} \mathbf{~ m}\end{array}$ \\
\cline { 2 - 7 } & Mean & STD & Mean & STD & Mean & STD \\
\hline Flex & $-6,6 \%$ & $17,7 \%$ & $-8,5 \%$ & $14,2 \%$ & $-6,6 \%$ & $26 \%$ \\
\hline One & $0,2 \%$ & $1,5 \%$ & $0,0 \%$ & $0,4 \%$ & $-4,9 \%$ & $8,2 \%$ \\
\hline iHealth & $-11,4 \%$ & $19,9 \%$ & $-0,8 \%$ & $2,4 \%$ & $-8,1 \%$ & $6,4 \%$ \\
\hline Vifit & $-10,3 \%$ & $11,7 \%$ & $-2,8 \%$ & $5,6 \%$ & $-9,2 \%$ & $4,3 \%$ \\
\hline Withings & $-1,3 \%$ & $2,0 \%$ & $-0,6 \%$ & $2,0 \%$ & $5,1 \%$ & $9,8 \%$ \\
\hline Jawbone & $-7,8 \%$ & $14,7 \%$ & $4,7 \%$ & $11,3 \%$ & $-7,2 \%$ & $21 \%$ \\
\hline Moves & $-7,2 \%$ & $25,2 \%$ & $-0,2 \%$ & $3,0 \%$ & $-5,6 \%$ & $1,4 \%$ \\
\hline \multicolumn{7}{|c|}{ TABLE V } \\
\hline
\end{tabular}

THE UPDATED ERROR RANGES FOR WALKING EXERCISES

\begin{tabular}{|c|c|c|}
\hline & Error range (min - max), \% \\
\hline Device type & Steps & Distance \\
\hline Accelerometer & $0,0-47,5$ & $1,0-41,2$ \\
\hline Accelerometer + GPS & $0,0-37,1$ & $3,8-7,4$ \\
\hline
\end{tabular}

TABLE VI

THE RELIABILITY FACTORS OF THE DEVICES

\begin{tabular}{|c|c|c|c|}
\hline Device & $\mathbf{D}_{\mathbf{s}}$ & $\mathbf{D}_{\mathbf{d}}$ & $\mathbf{D}$ \\
\hline Flex & 0,781 & 0,879 & 0,830 \\
\hline One & 0,990 & 0,968 & 0,979 \\
\hline iHealth & 0,830 & 0,860 & 0,845 \\
\hline Vifit & 0,853 & 0,896 & 0,874 \\
\hline Withings & 0,971 & 0,964 & 0,968 \\
\hline Jawbone & 0,818 & 0,891 & 0,854 \\
\hline Moves & 0,826 & 0,846 & 0,836 \\
\hline
\end{tabular}

$$
\begin{aligned}
& \left.D_{s}=0.5 \cdot \mid\left(1-E S_{\text {mean }, 100}\right) \cdot\left(1-E S_{\text {mean }, 1000}\right)\right] \\
& +0.5 \cdot\left[\left(1-E S_{S T D, 100}\right) \cdot\left(1-E S_{S T D, 1000}\right)\right] \\
& D_{d}=0.5 \cdot\left[\left(1-E D_{\text {mean }, 1000}\right)\right]+0.5 \cdot\left[\left(1-E D_{S T D, 1000}\right)\right]
\end{aligned}
$$

Where:

$D_{s}$ : reliability of step counting for physical activity devices;

$D_{d}$ : reliability of distance estimation for PA devices;

$E S_{\text {mean, } 100}$ : mean of error in step count in $100 \mathrm{~m}$ walk;

$E S_{\text {mean, } 1000}$ : mean of error in step count in $1000 \mathrm{~m}$ walk;

$E D_{\text {mean, } 1000}:$ mean of error in distance estimation in $1000 \mathrm{~m}$ walk;

$E S_{S T D, 100}$ : STD of error in step count in $100 \mathrm{~m}$ walk;

$E S_{S T D, 1000}:$ STD of error in step count in $1000 \mathrm{~m}$ walk;

$E D_{S T D, 1000}$ : STD of error in distance estimation in $1000 \mathrm{~m}$ walk. 
TABLE VII

PERSONAL FACTORS INVESTIGATION

\begin{tabular}{|c|c|c|c|c|c|c|c|c|c|}
\hline \multicolumn{2}{|c|}{ Moves } & $\mathbf{P 1}$ & $\mathbf{P 2}$ & P3 & $\mathbf{P 4}$ & P5 & P6 & P7 & P8 \\
\hline \multirow{4}{*}{$\begin{array}{l}\text { Daily Walking Speed ( } V_{\text {daw }} \\
\text { (m/s) }\end{array}$} & MAX & 0.98 & 1.19 & 1.10 & 1.00 & 1.50 & 1.58 & 1.09 & 1.15 \\
\hline & MIN & 0.50 & 0.29 & 0.69 & 0.51 & 0.69 & 0.82 & 0.50 & 0.68 \\
\hline & AVER & 0.68 & 1.00 & 0.99 & 0.85 & 1.26 & 1.09 & 0.84 & 1.03 \\
\hline & STDEV & 0.14 & 0.13 & 0.10 & 0.10 & 0.16 & 0.17 & 0.19 & 0.12 \\
\hline \multirow{4}{*}{ Walking Cadence (steps/s) } & MAX & 1.86 & 1.85 & 1.78 & 1.44 & 1.95 & 1.84 & 1.82 & 1.91 \\
\hline & MIN & 0.67 & 1.13 & 1.12 & 0.82 & 1.35 & 1.13 & 0.67 & 1.15 \\
\hline & AVER & 1.24 & 1.54 & 1.50 & 1.18 & 1.60 & $\mathbf{1 . 5 3}$ & 1.31 & 1.54 \\
\hline & STDEV & 0.29 & 0.15 & 0.16 & 0.10 & 0.14 & 0.14 & 0.27 & 0.21 \\
\hline \multicolumn{6}{|c|}{ Fitbit } & & & & \\
\hline \multirow{4}{*}{ DAPS (m/s) } & MAX & 2.17 & 2.18 & 2.40 & 1.93 & & & & \\
\hline & MIN & 0.17 & 0.55 & 0.62 & 1.82 & & & & \\
\hline & AVER & 1.72 & 1.88 & 2.07 & 1.88 & & & & \\
\hline & STDEV & 0.62 & 0.28 & 0.40 & 0.08 & & & & \\
\hline \multirow{4}{*}{ Active Speed $(\mathrm{m} / \mathrm{s})$} & MAX & 1.42 & 1.53 & 1.82 & 1.30 & & & & \\
\hline & MIN & 1.22 & 1.10 & 1.25 & 1.22 & & & & \\
\hline & AVER & $\mathbf{1 . 3 0}$ & 1.27 & 1.50 & 1.25 & & & & \\
\hline & STDEV & 0.05 & 0.12 & 0.13 & 0.06 & & & & \\
\hline \multirow{4}{*}{ Moderate Speed $(\mathbf{m} / \mathbf{s})$} & MAX & 0.67 & 0.65 & 0.65 & 0.50 & & & & \\
\hline & MIN & 0.33 & 0.41 & 0.46 & 0.47 & & & & \\
\hline & AVER & 0.57 & 0.52 & 0.56 & 0.48 & & & & \\
\hline & STDEV & 0.08 & 0.05 & 0.05 & 0.03 & & & & \\
\hline \multirow{4}{*}{ Slightly Speed $(\mathbf{m} / \mathbf{s})$} & MAX & 0.18 & 0.17 & 0.18 & 0.15 & & & & \\
\hline & MIN & 0.13 & 0.13 & 0.13 & 0.13 & & & & \\
\hline & AVER & 0.16 & 0.14 & 0.15 & 0.14 & & & & \\
\hline & STDEV & 0.01 & 0.01 & 0.01 & 0.001 & & & & \\
\hline \multicolumn{10}{|c|}{ Pearson Correlation } \\
\hline \multirow{4}{*}{ 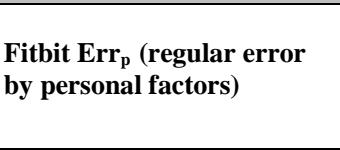 } & DAPS & 0.74 & 0.12 & 0.380 & 0.23 & & & & \\
\hline & Active & -0.16 & -0.16 & -0.20 & -0.14 & & & & \\
\hline & Moderate & -0.07 & 0.47 & 0.09 & 0.21 & & & & \\
\hline & Slightly & 0.17 & -0.07 & 0.07 & 0.12 & & & & \\
\hline \multirow{2}{*}{$\begin{array}{l}\text { Moves } \text { Err }_{p} \text { (regular error } \\
\text { by personal factors) }\end{array}$} & DAPS (Daily Walking Speed) & 0.51 & 0.17 & 0.50 & 0.38 & 0.14 & 0.03 & 0.59 & 0.23 \\
\hline & Walking Cadence & 0.19 & 0.12 & 0.09 & 0.19 & 0.11 & -0.07 & 0.44 & 0.11 \\
\hline
\end{tabular}

Following equation (3), the calculated reliability factors (with the weight $k=0,5$ ) are presented in Table VI. We observe that One is the most reliable while Withings shows only slightly lower performance. Moves (GPS+Accelerometer equipment) performs similarly to Accelerometer only trackers worn on the wrist.

\section{Personal Factor}

In terms of the definition of DAPS in LPAV-IoT model, a person's physical fitness can be represented by a walking speed related score. Moves does not classify the intensity of physical activity regarding the walking speed, so its DAPS is equal to Average Daily Walking Speed. Fitbit Flex physical activity data has been classified into the intensity of four types as so DAPS and its related walking speeds are measured. Each person has different physical activity characteristics, such as walking speed. The issue here is that individual physical characteristics will impact the accuracy of collected raw data. We measure the parameters like MAX, MIN, AVER and STDEV of users historical raw data. In order to ensure the diversity of data, we allow MHA platform to be used by 28 users from 4 project partners ( 2 universities, 2 companies) within the EU. We collect daily physical activity (Steps, Distance and Calories) of these 28 users over 6 months by 3 types of wearable devices of recorders (Withings, One and Moves). All these users are professionals with age in the range of 20-60 years old. We choose 2 persons physical activity data from each partner as representations, and in total 8 persons for investigation, as shown in Table.7. The features are:

- In Moves, 8 people average walking speed is $0.69 \mathrm{~m} / \mathrm{s} \sim 1.26$ $\mathrm{m} / \mathrm{s} ; 8$ people average step speed is $1.18 \mathrm{step} / \mathrm{s} \sim 1.60 \mathrm{step} / \mathrm{s}$; the figure using Moves segment (minute-by-minute) data is slightly lower than Moves summary (daily).

- In Flex, 4 people DAPS is $1.72 \mathrm{~m} / \mathrm{s} \sim 2.07 \mathrm{~m} / \mathrm{s}$; active average step speed is $1.30 \mathrm{~m} / \mathrm{s} \sim 1.50 \mathrm{~m} / \mathrm{s}$; moderate average step speed is $0.48 \mathrm{~m} / \mathrm{s} \sim 5.07 \mathrm{~m} / \mathrm{s}$; slightly average step speed is $0.14 \mathrm{~m} / \mathrm{s} \sim 0.16 \mathrm{~m} / \mathrm{s}$. Each person has different physical activity, but their daily speed or DAPS are in a similar range.

Regarding the international standard of human walking cadence and speed, female walking is roughly 1.95 steps/s in cadence and $1.85 \mathrm{~m} / \mathrm{s}$ in speed; male waking is about 1.95 steps/s in cadence, and his average speed is $1.43 \mathrm{~m} / \mathrm{s}$. It appears that both Flex and Moves underestimate users' walking speed. Pearson Correlation Coefficient ( $r$ ) is used for measuring the relationship between DAPS or walking speeds and Err , as shown in Table III. The Pearson Correlation results reflect variability among individual subjects, for instance, in One (DAPS vs Err), the physical fitness of Subject P1 may have a strong relationship with irregular errors, which gives a value up to 0.73 ; but for subjects $\mathrm{P} 2$ and $\mathrm{P} 3$, this relationship has only a value lower to 0.12. Similarly, in Moves, the value of Pearson Correlation differs among subjects in the range 0.173-0.589. 
The findings indicate that differences in physical fitness of personal factors will not generate significant regular errors in physical activity data. The rules are: a) Pearson Correlation Coefficient (r) between Daily Speed and Daily Steps for individual is diverse. b) No strong impact of daily speed or MAPS on daily steps. While each subject has different physical activity ability, but their speed or MAPs are within a range, and no correlation with daily steps was observed. c) Personal factors (for normal people) will not generate significant errors in physical activity data.

\section{Geographic Factors}

Following validation strategy in Section III.D, the impact of geographic factor on irregular uncertainties is estimated by using empirical analysis methods on observed data of a small group of daily physical activity. We analysed Day-of-Week differences in this dataset including all three devices (Fitbit One, Moves and Withings) for both groups and individual. Fig.4 and Fig.5 illustrate the distribution of Day-of-Week difference on group and individual daily physical activity. In Fig.5, the lines of (P1_m,...P7_m) represent Moves users; the lines of $\left(\mathrm{P} 1 \_\mathrm{f}, \ldots, \mathrm{P} 3 \_\mathrm{f}\right)$ represents Fitbit One users; and the lines of (P4_w, P5_w) represent Withings users. Also, Moves provide time based walking segments data, we conduct the distribution of Time-of-Day difference on group based physical activity in Fig.6. In Fig.6, the physical activity at certain time-slot in a group of 7 users is summed as Distance, Steps and Durations. The features of this data are:

- For Day-of-Week difference, a similar trend line of group physical activity occurs in three devices. It shows that daily step appears stable in weekdays but decreases dramatically on weekend.

- The trend line of individual physical activity is fluctuated widely, but approximately follows the same trend of group physical activity.

- For Time-of the Day difference, the highest intensity of physical activity occurs from $7 \mathrm{am}$ to $10 \mathrm{am}$. Then the intensity of physical activity keeps stable and slightly decreases in the Afternoon. At the night from 11 pm-12 pm, the intensity of physical activity increases bit. But it may be because users use their smartphone before sleep.

The rules are concluded below: a) People normally have stable physical activity in working day, but have much less physical activity on Sunday. b) People normally have an intensive physical activity in the morning session (7-10 am), and have moderate physical activity in other time of the day.

\section{Case Study and Performance Evaluation}

In this section, we discuss the performance evaluation of LPAV-IoT model in a case study on MHA platform [38], which is an IoT based healthcare project. MHA platform enables users to record, store and visualize their multi-dimensional health data by connecting wearable devices or mobile apps, like Fitbit Flex, Moves, Withings, Twitter and Facebook. The criteria of verifying LPAV-IoT are based on their performance of using its rules for: irregular uncertainties filtering, reliability estimation and model adaptivity. We collected the empirical dataset by using MHA platform. The dataset includes 12 months long daily physical activity of 28 persons from 4 project partners ( 2

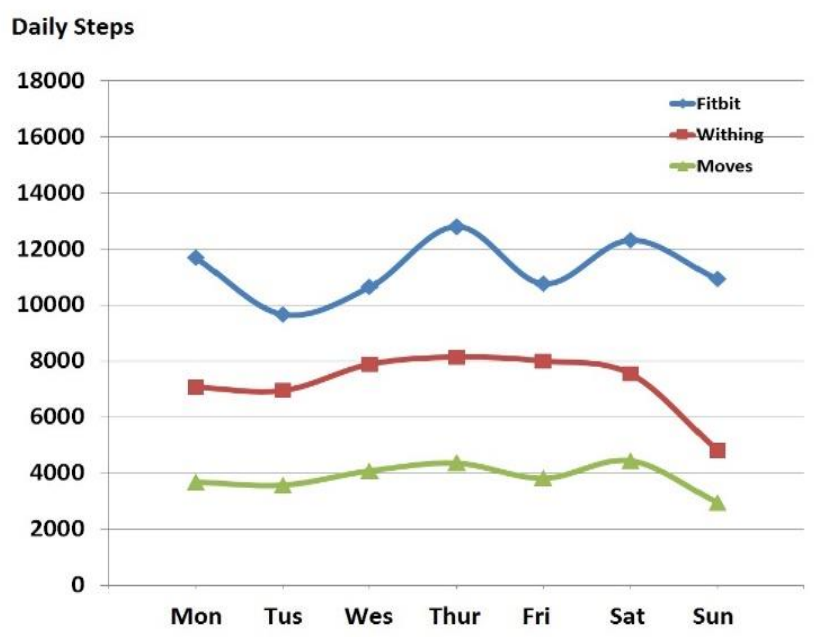

Fig. 4 Distribution of Day-of-Week difference on group based daily physical activity

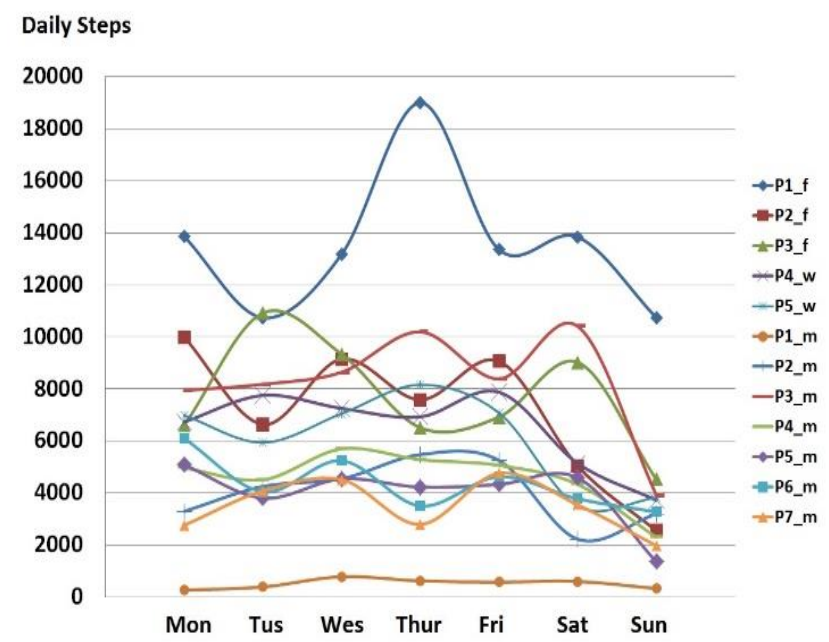

Fig. 5 Distribution of Day-of-Week difference on individual based daily physical activity

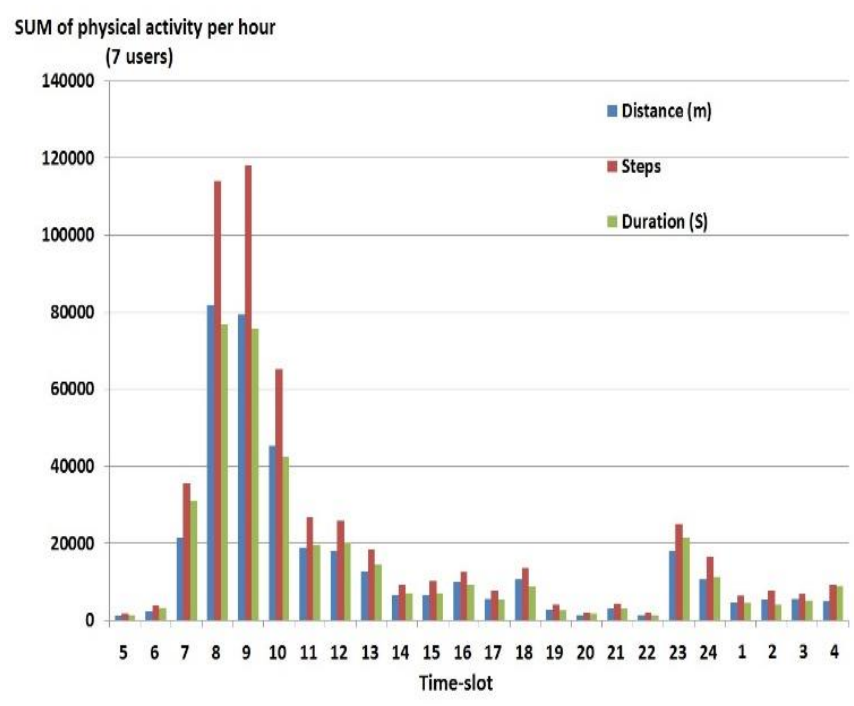

Fig. 6 Distribution of Time-of-Day difference on group based physical activity 
universities, 2 companies) within EU acquired with three devices: Moves was used by 28 users for 12 months; Flex was used by 10 users for 12 months; Withings was used by 8 users for 6 months. These people are healthy in the age range of 20-60 years. The evaluation methodology for verifying the efficiency of proposed model will interview the participants, and collect feedbacks on reflecting users' experiences on physical activity uncertainties through different devices. The feedbacks are used as a standard benchmark to compare the correctness of model.

\section{A. Filtering Irregular Uncertainties (UI)}

In order to validate the accuracy of identifying IU, we follow equation (4) with a confidence interval of $95 \%$ to filter data from three different devices. We use the values $(130,1784,884)$ of threshold parameter Ts respectively in Moves, One and Withings, for filtering incorrect daily steps data. The results are shown in Table VIII.

TABLE VIII

REMOVING IRREGULAR UNCERTAINTIES BY LPAV-IOT

\begin{tabular}{|c|c|c|c|}
\hline & Moves & Flex & Withings \\
\hline Ts Daily Steps & 130 & 1784 & 1267 \\
\hline $\mathrm{T}_{\mathrm{Y}}$ DAPS Speed (m/s) & 0.5 & 1.50 & NA \\
\hline Total number of People & 14 & 5 & 3 \\
\hline Percentage of people with IU & $43 \%$ & $100 \%$ & $100 \%$ \\
\hline Number of IU occurrence & 40 & 17 & 8 \\
\hline IU confirmed by User & 40 & 15 & 6 \\
\hline $\begin{array}{c}\text { Average number of IU occurrence per } \\
\text { person (User Feedback) }\end{array}$ & 6.6 & 5.4 & 2.7 \\
\hline Accuracy of identifying IU (95\%) & $100 \%$ & $88.2 \%$ & $75 \%$ \\
\hline
\end{tabular}

Moves has much lower threshold parameters of Daily Steps and DAPS speed than Flex and Withings which are 130 and 0.5 $\mathrm{m} / \mathrm{s}$ respectively (Table VIII). This is because Moves has larger device uncertainties than Withings and Flex as we observed in section IV.C. Thus the GPS and smartphone internal sensors based App is not as accurate as accelerometer only based wrist wearable device. In terms of percentage of people having IU, Moves is much lower than Withings and Flex. It is probably because most of uncertainties from Moves have been classified into regular uncertainties, so its irregular uncertainties became less than for other two devices Withings and Flex. However, for average IU occurrence per subject, Moves has higher performance than other two devices (Table VIII). The accuracy of identifying IU appears that on the condition with a confidence interval of $95 \%$, the related value of threshold parameter $T s$ can successfully filter irregular uncertainty in Moves. So Moves have the best IU identification accuracy up to $100 \%$, which means that the incorrect daily steps detected by LPAV-IoT model in Moves have been all approved by users. Flex and Withings have accuracy up to $88.2 \%$ and $62.5 \%$ respectively, which implies that some correct daily steps are eliminated by LPAV-IoT model.

The increase of confidence interval impacts on filtering accuracy of IU in LPAV-IoT, as shown in Table IX. If we increase the confidence interval up to $98 \%$, and recalculate threshold parameters, the accuracy of identifying IU of three devices would increase to $100 \%$. But, a noticeable issue here is that if we increase the confidence interval, some IU might be ignored and put into the procedure of dealing with regular uncertainties in LPAV-IoT model. Similarly, in Moves, a high accuracy of identifying IU does not mean all the IU have been removed, probably some of IUs are considered as regular uncertainties in LPAV-IoT model.

TABLE IX

REMOVING IRREGULAR UNCERTAINTIES BY LPAV-IOT WITH INCREASING CONFIDENCE INTERVAL

\begin{tabular}{|c|c|c|c|}
\hline & Moves & Flex & Withings \\
\hline Total number of People & 14 & 5 & 3 \\
\hline Percentage of people with IU & $43 \%$ & $100 \%$ & $100 \%$ \\
\hline Number of IU occurrence & 40 & 17 & 8 \\
\hline $\begin{array}{c}\text { Average number of IU occurrence per } \\
\text { person (User Feedback) }\end{array}$ & 6.6 & 5.4 & 2.7 \\
\hline Accuracy of identifying IU (95\%) & $100 \%$ & $88.2 \%$ & $75 \%$ \\
\hline Accuracy of identifying IU (96\%) & $100 \%$ & $92.4 \%$ & $87.5 \%$ \\
\hline Accuracy of identifying IU (97\%) & $100 \%$ & $96.5 \%$ & $87.5 \%$ \\
\hline Accuracy of identifying IU (98\%) & $100 \%$ & $100 \%$ & $100 \%$ \\
\hline
\end{tabular}

\section{B. Reliability Estimation}

For validating reliability indicator of regular RU, we follow the strategies of LPAV-IoT model and equations in Section IV to process the above dataset for getting average figures of the group of 14 people. Then we choose the data of one person (P1 in Table VII) who has three devices for estimating reliability indictor. The feedback from this person will assess the efficiency of our proposed reliability indictor. The criteria of interpreting the feedbacks contain five levels of agreement (Almost perfect, Substantial, Moderate, Fair, Slight). The results are shown in Table $X$.

$$
\text { TABLE X }
$$

REGULAR UNCERTAINTIES INDICATOR BY LPAV-IOT

\begin{tabular}{|c|c|c|c|}
\hline $\begin{array}{c}\text { Reliability } \\
\text { Indicator }\end{array}$ & Moves & Flex & Withings \\
\hline D & $83.6 \%$ & $83.0 \%$ & $96.8 \%$ \\
\hline P & $87.6 \%$ & $96.7 \%$ & $95.6 \%$ \\
\hline $\mathbf{E}$ & $78.6 \%$ & $83.4 \%$ & $87.4 \%$ \\
\hline R & $\mathbf{5 7 . 5 \%}$ & $\mathbf{6 6 . 7 \%}$ & $\mathbf{8 0 . 9 \%}$ \\
\hline User Feedback & Moderate & Substantial & Almost perfect \\
\hline
\end{tabular}

Table $\mathrm{X}$ reflects that using the regular reliability indicator of LPAV-IoT model, the reliability estimation of collected physical activity data by three devices were approximately following the users' feedback. The data from Moves is estimated as reliability of $57.7 \%$, and user believes this data are moderately accurate. The data from Flex and Withings are both more reliable than Moves regarding user's feedback. Especially, Withings is recognized by user as "almost perfect", which has a reliability value up to $80.9 \%$. Flex is slightly less reliable than Withings, it is mainly from the difference of device factors. Above figures imply that the proposed reliability indicator of LPAV-IoT model can be used as a quantitative analysis tool to estimate the reliability of personalized physical activity data collected from an IoT environment.

\section{Model Adaptivity}

For validating the adaptivity of LPAV-IoT model, we consider the whole group of 14 subjects as one group due to the similar professions and backgrounds. We estimate the change of daily steps $T_{\mathrm{s}}$ and DAPS with different periods (from 1 month to 12 months) with a confidence interval of $95 \%$. The results are shown in Fig.7 and 8. Fig.7 shows the parameter Daily Steps as the function of time period duration. The value of this parameter is lower for shorter time periods than for longer time periods. The value of this parameter also varies with different devices. For Moves and Withings, the value of 
this parameter over different periods is slightly growing, but for Fitbit, this parameter dramatically increases after 6 months. This effect may be influenced by the setting of confidence interval. Fig. 8 shows little variation of parameter DAPS in the LPAV-IoT model when time period duration is changed. There are some mirror fluctuations of DAPS on both Moves and Fitbit. But in a long term, the value of DAPS is quite stable, which indicates that personal physical fitness does not have significant changes within this group of 14 people.

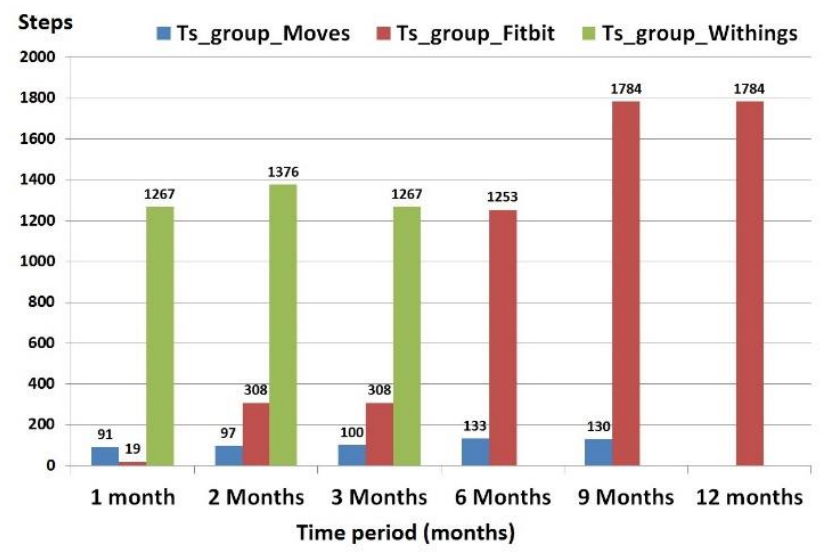

Fig. 7 Average of daily steps as the function of time period duration

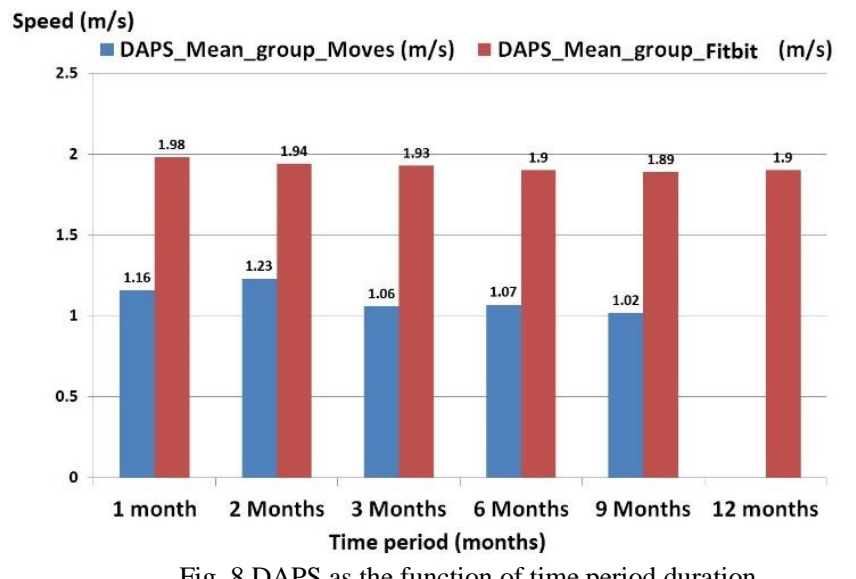

Fig. 8 DAPS as the function of time period duration

\section{DISCUSSION AND FUTURE WORK}

While LPAV-IoT model addresses a pioneered investigation on effectively validating lifelogging physical activity data for IoT enabled personalized health systems, it has several issues to discuss and consider in future.

\section{A. Extendibility}

Current LPAV-IoT model has mainly considered the impacts of personal, device and geographic factors on the validity of lifelogging physical activity. But in a practical IoT ecosystem, there are other issues influencing the measures of lifelogging physical activity, e.g. social events in calendar, diverse subjects of daily activities. LPAV-IoT model is capable to be extended by either detailing a key impacting issue into several specific items or adding new representative blocks for rising issues, for supporting the quantified investigations of their impacts. For instance, social events in calendar like bank holiday in the UK is treated as a specific item in Timeline dimension; longitudinal data analysis methodology in Fig. 2 is directly applied into this item for conducting validation rules. The diverse subjects of daily activities require adding a new block "activity subject" into LPAV-IoT model. The data analysis methodology in this block will include typical classification approaches in activity recognition, e.g. decision tree. The conducted validation rules from new blocks may be not directly useable in the reliability equations in LPAV-IoT model, but will be benefit to users for removing uncertainties of physical activities on specific cases. Similarly, other new considerable factors can be extended into our proposed LPAV-IoT model.

\section{B. Human-in-the-loop}

LPAV-IoT model is designed as Human-in-the-loop since the validation rules is supposed to be adaptively altered regarding the properties of its human factor, like age, gender, group or interaction, etc. For instance, section V.C gives a performance comparison of individual and group population (14 persons with similar professions and backgrounds) on removing irregular uncertainties. We estimate the change of daily steps $\mathrm{T}_{\mathrm{s}}$ and DAPS with different periods (from 1 month to 12 months) with a confidence interval of $95 \%$. The results shown in Fig.7 and 8 indicate that the rules of LPAV-IoT model will be altered in terms of different setting of human factors. However, this experiment only deals with a nature increment of life-logging physical activity on timeline and population dimensions. It is not a strict performance evaluation of human-in-the-loop in the proposed model by considering a human interaction with model. The involvement of collecting user feedbacks as a step of the validation algorithm is not hard to be implemented in the model, but requires a long period of time on re-designing experimental strategies and collecting relevant life-logging data. Thus, it will be put as one of key future works in LPAV-IoT model, which is to continue a formal human-in-the-loop validation of the model by involving users' feedbacks for updating validation rules.

\section{Limitations}

First, the scalability of LPAV-IoT model for dealing with increased volume and types of health data is not yet considered in this paper. In practical IoT enabled personalized healthcare environment, personal health information will be a life-long collection, also include other medical data, such as ECG or blood pressure, etc. While LPAV-IoT model can be extended into improving accurate measures of physical activity related health data, like calories estimation, the practical efficiency on multi-type health data in a long term collection needs a further evaluation. Second, the evaluation of data validation efficiency and regular uncertainty indicator for LPAV-IoT model is subject to only few users' feedbacks. The standardized criteria of judging correctness and efficiency of LPAV-IoT model on removing and estimating uncertainties requires more users' feedbacks. Also, for different targeted groups, the adaptability of LPAV-IoT model needs to be verified by more users.

\section{Practical Value}

LPAV-IoT model provides a pioneered investigation approach for improving the validity of lifelogging physical activity in an IoT environment. While lifelogging techniques have been seen as a hot topic in research in the last twenties years, it recently becomes more accessible and practically significant with the 
recent prevalence of mobile devices connecting in IoT systems. In the healthcare field, due to significant population aging in the coming decades, IoT enabled technology is evolving healthcare from conventional hub based system to personalised healthcare system. The successful utilization of LPAV-IoT model into practical will enable more accurate measure and monitoring of daily physical activity with low cost devices, further lead to faster and safer preventive care for chronic diseases.

While LPAV-IoT model has above further future work, we believe that the benefit of LPAV-IoT model outweighs its limitations. LPAV-IoT model has provided a new approach to validate physical activity data in an IoT environment, also has been verified by a rich set of personal health data in real experiments. The research outcome is extremely valuable and benefit.

\section{CONCLUSIONS}

In this paper, a rule based adaptive physical activity validation model, LPAV-IoT, is proposed for eliminating irregular uncertainties and estimating data reliability in an IoT enabled personalized healthcare environment. It specifies four layers and three modules for evaluating the factors impacting the validity of lifelogging physical activity. The validation rules are represented by defining a set of uncertainty threshold parameters and reliability indicators, which are initiated by historical raw data and adaptively updated regarding the needs of an IoT enabled personalized healthcare system. Following this model, a case study on an IoT enabled healthcare platform MHA [38] connecting three state-of-the-art wearable devices and mobile apps was carried out. The results reflect that LPAV-IoT is an efficient, adaptive and extendable solution for the validation of IoT environment based physical activity data.

\section{REFERENCES}

[1] K. Kang, Z. B. Pang, L. D. Xu, L. Y. Ma and C. Wang, "An interactive trust model for application market of the internet of things," IEEE Trans. Ind.Informat, vol. 10, no. 2, pp. 1516-1526, Feb, 2014.

[2] H. R. Yan, Y. Zhang, Z. B. Pang and L. D. Xu, "Superframe planning and access latency of slotted MAC for industrial WSN in IoT environment," IEEE Trans. Ind. Informat, vol. 10, no. 2, pp. 1242-1251, Feb, 2014.

[3] P. Yang, W. Wu, M. Moniri and C. C. Chibelushi, "Efficient object localisation using sparsely distributed passive RFID tags," IEEE Trans. Ind. Electron, vol. 10, no. 2, pp. 1443-1451, Dec, 2013.

[4] L. H. Jiang, L. D. Xu, H. M. Cai, Z. H. Jiang, F. L. Bu and B. Y. Xu, "An IoT-Oriented data storage framework in cloud computing platform," IEEE Trans. Ind. Informat, vol. 10, no. 2, pp. 1443-1451, Feb, 2014.

[5] W. He, G. J. Yan and L. D. Xu, "Developing vehicular data cloud services in the IoT environments," IEEE Trans. Ind. Informat, vol. 10, no. 2, pp. 1587-1595, Feb, 2014.

[6] P. Yang and W. Wu, "Efficient particle filter localisation algorithm in dense passive RFID tags environment," IEEE Trans. Ind. Electron, vol. 61, no. 10, pp. 5641-5661, Oct, 2014.

[7] Z. M. Bi, L. D. Xu and C. G. Wang, "Internet of Things for enterprise systems of modern manufacturing," IEEE Trans. Ind. Informat, vol. 10 no. 2, pp. 1537-1546, Feb, 2014.

[8] H. M. Cai, L. D. Xu, B. Y. Xu, C. Xie, S. J. Qin and L. H. Jiang, "IoT based configurable information service platform for product lifecycle management," IEEE Trans. Ind. Informat, vol. 7, no. 2, pp. 1558-1567, Feb, 2011.
[9] C. G. Wang, Z. M. Bi and L. D. Xu, "IoT and cloud computing automation of assembly modeling systems," IEEE Trans. Ind. Informat, vol. 10, no. 2, pp. 1426-1434, Feb, 2014.

[10] L. Li, S. C. Li and S. S. Zhao, "OoS-aware scheduling of services-oriented internet of things," IEEE Trans. Ind. Informat, vol. 10, no. 2, pp. 1497-1505, Feb, 2014.

[11] X. R. Zheng, P. Martin, K. Brohman and L. D. Xu, "Cloudqual: A quality model for cloud services," IEEE Trans. Ind. Informat, vol. 10, no. 2, pp. 1527-1536, Feb, 2014.

[12] S. Amendola, R. Lodato, S. Manzari, C. Occhiuzzi and G. Marrocco, "RFID technology for IoT-based personal healthcare in smart spaces," IEEE Internet of Things Journal, vol. 1, no. 2, pp. 144-152, 2014.

[13] X. Li, R. X. Lu, H. Liang, X. M. Shen, J. M. Chen and X. D. Lin, "Smart community: An internet of things application," IEEE communication magazine, vol. 49, no. 11, pp. 68-75, 2011.

[14] Y. Fan, Y. Yin, L. Xu, Y. Zheng and F. Wu, "Iot based smart rehabilitation system," IEEE Trans. Ind. Informat, vol. 10, no. 2, pp. 1568-1577, Feb, 2014.

[15] G. Yang, L. Xie, M. Mantysalo, X. L. Zhou, Z. B. Pang, L. D. Xu, S. Kao-Walter, Q. Chen and L. R. Zheng, "A health-IoT platform based on the integration of intelligent packaging, unobstrusive bio-sensor, and intelligent medicine box," IEEE Trans. Ind. Informat, vol. 10, no. 4, pp. 2180-2192, Nov, 2014.

[16] B. Y. Xu, L. D. Xu, H. M. Cai, C. Xie, J. Y. Hu and F. L. Bu, "Ubiquitous data accessing method in IoT-based information system for emergency medical services," IEEE Trans. Ind. Informat, vol. 10, no. 2, pp. 1578-1587, May, 2014.

[17] D. He and S. Seadally, "An analysis of RFID authentication schemes for internet of things in healthcare environment using elliptic curve cryptography," IEEE Internet of Things Journal, vol. 2, no. 1, pp. 72-83, 2015.

[18] F. Daniel, F. Casati, P. Silveira, M. Verga and M. Nalin, "Beyond health tracking: A personal health and lifestyle platform," IEEE Internet Computing, vol. 15, no. 4, pp. 14-22, 2011.

[19] G. Sebestyen, A. Hangan, S. Oniga and Z. Gal, "eHealth solutions in the context of Internet of Things," in IEEE Conference of Automation, Quality and Testing, Robotics, Cluj-Napoca, 2014.

[20] R. S. Istepanian, S. Hu, N. Y. Philip and A. Sungoor, "The potential of internet of m-health things "m_IoT" for non-invasive glucose level sensing," in The 33rd Annual International Conference of the IEEE EMBS, Boston, Massachusettes, USA, 2011.

[21] R. Paradiso, G. Loriga and N. Taccini, "A wearable healthcare system based on knitted integrated sensors," IEEE Trans. Info. Tech. Bio, vol. 9, no. 3, pp. 337-344, 2005.

[22] J. Parkka, M. Ermes, P. Korpipaa, J. Mantyjarvi, J. Peltola and I. Korhonen, "Activity classification using realistic data from wearable sensors," IEEE Trans. Info. Tech. Bio, vol. 10, no. 1, pp. 119-128, 2006.

[23] M. Ermes, J. Parkka, J. Mantyjarvi and I. Korhonen, "Detection of daily activities and sports with wearable sensors in controlled and uncontrolled conditions," IEEE Trans. Info. Tech. Bio, vol. 12, no. 1, pp. 20-26, 2008.

[24] H. Ghasemzadeh and R. Jafari, "Physical movement monitoring using body sensor networks: a phonological approach to construct spatial decision trees," IEEE Trans. Ind. Informat, vol. 7, no. 1, pp. 66-77, Feb, 2011.

[25] D. Curone, A. Tognetti, E. L. Secco, G. Anania, N. Carbonaro, D. DeRossi and G. Magenes, "Heart rate and accelerometer data fusion for activity assessment of rescuers during emergency interventions," IEEE Trans. Info. Tech. Bio, vol. 14, no. 3, pp. 702-710, 2010.

[26] J. Qi, P. Yang, D. Fan and Z. Deng, "A Survey of Physical Activity Monitoring and Assessment Using Internet of Things Technology", 2015 IEEE International Conference on Pervasive Intelligence and Computing, pp. 2353-2358, Liverpool, UK, Oct, 2015.

[27] L. Bao and S. S. Intille, "Activity recognition from user-annotated acceleration data," Pervasive Computing, vol. 3001, pp. 1-17, 2004.

[28] J. Lester, T. Choudhury and G. Borriello, "A practical approach to recognizing physical activity," Pervasive Computing, vol. 3968, pp. 
1-16, 2006.

[29] F.F.Guo, Y. Li, M.S. Kankanhalli and M.S.Brown, "An evaluation of wearable activity monitoring devices", Proceedings of the $1^{\text {st }}$ ACM International Workshop on Personal Data meets Distributed Multimedia, ppp. 31-32, 2013, Newyork.

[30] P. Yang, M. Hanneghan, J. Qi, Z. Deng, F. Dong and D. Fan, "Improving the Validity of Lifelogging Physical Activity Measures in an Internet of Things Environment", 2015 IEEE International Conference on Pervasive Intelligence and Computing, pp. 2309-2314, Liverpool, UK, Oct, 2015.

[31] L. Atallah, B. Lo, R. King and G. Z. Yang, "Sensor positioning for activity recognition using wearable accelerometers," IEEE Trans. Bio. Cir \& Sys, vol. 5, no. 4, pp. 320-329, 2011.

[32] G. Y. Xiao, J. Z. Guo, L. D. Xu and Z. G. Gong, "User interoperability with heterogeneous IoT devices through transformation," IEEE Trans. Ind. Informat, vol. 7, no. 2, pp. 1486-1496, 2011.

[33] N. Alshurafa, W. Y. Xu, J. Liu, M. C. Huang, B. Mortazavi, C. K. Roberts and M. Sarrafzadeh, "Designing a robust activity recognition framework for health and exergaming using wearable sensors," IEEE Journal of Bio \& Health Info, vol. 18, no. 5, pp. 1636-1646, 2014.

[34] S. D. Herrmann, E. M. Snook, M. Kang, C. B. Scott, M. G. Mack, T. P. Dompier and B. G. Ragan, "Development and validation of a movement activity in physical space score as a functional outcome measure," Arch. Phys. Med. Rehabil, vol. 18, no. 5, pp. 1636-1646, 2011.

[35] E. G. Spanakis, D. Kafetzopoulos, P. Yang, K. Marias, Z. K. Deng, M. Tsikanakis, F. Dong and V. Sakkalis, "MyHealthAvatar: Personalized and empowerment health services through internet of things

technologies," in EAI 4th International Conference on Wireless Mobile Communication and Healthcare, Athens, 2014.

[36] L. Gargasas, V. Jurkonis, L. Bikuliene, A. Zvironiene and S. Daukantas, "Functional state evaluation system with distributed intellect for elderly and disabled persons," Technologies for Computer Control, vol. 13, pp. 57-52, 2012.

[37] "CARRE" CARRE project, 2013. [Online]. Available: http://www.carre-project.eu /. [Accessed 10 Oct 2014].

[38] "MHA," MHA project, 2013. [Online]. Available: http://www.myhealthavatar.eu/. [Accessed 10 Oct 2014].

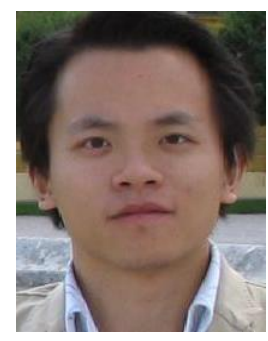

Po Yang is a Senior Lecturer in the department of Computing Science at Liverpool John Moores University, UK. $\mathrm{He}$ received the $\mathrm{PhD}$ degree in Electronic Engineering from the University of Staffordshire, UK, in 2010, and the M.Sc. degree in Computer Science from the University of Bristol, UK, in 2006, and the B.Sc. degree in Computer Science from Wuhan University, China, in 2004. Dr Yang has a wide range of research interests, including Internet of Things, RFID and indoor localisation, Pervasive Health, Image Processing, GPU and Parallel Computing. He holds a strong tracking of high quality publications and research experiences. He has published over 40 papers.

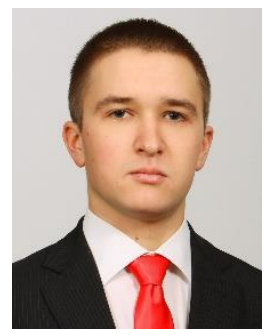

Dainius Stankevicius received the B.Sc. degree in electronics engineering and the M.Sc. degree in biomedical engineering from Kaunas University of Technology, Kaunas, Lithuania, in 2014 and 2016, respectively. $\mathrm{He}$ is currently with the Biomedical Engineering Institute at Kaunas University of Technology where he is a researcher. His research interests are unobtrusive systems for long-term health monitoring, and real-time signal processing algorithms for embedded applications.

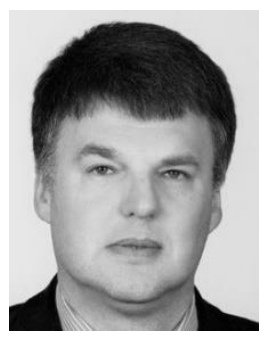

Vaidotas Marozas received the B.Sc., M.Sc., and Ph.D. degrees in electrical engineering from Kaunas University of Technology (KTU), Kaunas, Lithuania, in 1993, 1995, and 2000, respectively. He is currently a Professor at Department of Electronics, Faculty of Electrical and Electronics Engineering and the Head of the Multimodal Biosignal Laboratory of Biomedical Engineering Institute, KTU. His research interests fall in the area of multimodal biomedical signal processing and embedded systems for biomedical monitoring: detection and characterization of cardiac arrhythmias in bioelectrical and biooptical signals, arterial stiffness estimation and monitoring, technologies for physical activity monitoring. Vaidotas Marozas has been principal investigator of several national and commercial projects. He has co-authored 18 publications in international peer-reviewed journals and several textbooks on biomedical engineering.

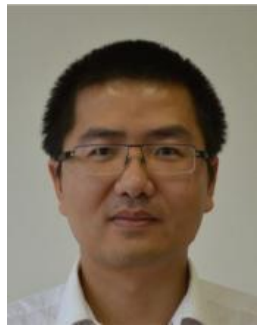

Zhikun Deng received his first degree in Mechanical Engineering and Automation from Beihang University in Beijing China 2004. And his MSc Degree in Computer Science from Bristol University UK, in 2007. And he is currently a $\mathrm{PhD}$ candidate in Computer Science at University of Bedfordshire. His main research areas are big data architecture, security and analysis.

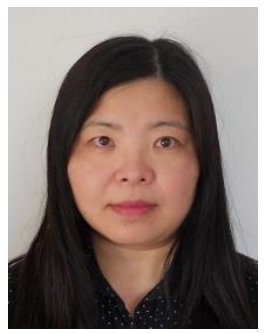

Enjie Liu received her first degree in Computer Science from Southwestern University, China in 1987. She received her $\mathrm{PhD}$ from Queen Mary, University of London in Telecommunication Networks in 2002. She joined in University of Bedfordshire in 2003, and currently is Reader in Network Applications and worked at Centre for Intelligent Data Analytics and Visualisation. She also had several years of experiences in industry before moved to academic. Her research interests include network protocols and data analytics for monitoring and predicting disease progressions for patients.

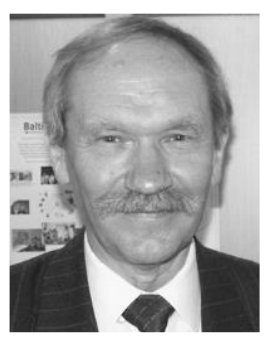

Arunas Lukosevicius received the Dipl.Eng., Ph.D., and Doctor Habilitus degrees from the Kaunas University of Technology (KTU), Kaunas, Lithuania, in 1964, 1976, and 1996, respectively, all in electronics. He is currently the Director of Biomedical Engineering Institute, KTU, and a Full Professor in the Department of Signal Processing, Faculty of Telecommunication and Electronics, KTU. His research interests include biosignal processing, wireless sensing of physiological processes, ultrasonic tissue characterization, and integration of smart monitoring devices in eHealth environment. 


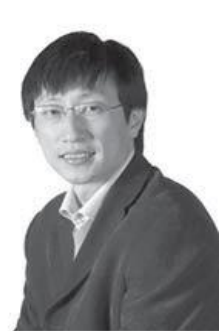

Feng Dong received the B.Sc., M.Sc., and $\mathrm{Ph} . \mathrm{D}$. degrees from Zhejiang University, Hangzhou, China. He is a Professor of visual computing. He joined as CCGV from Brunel University, Middlesex, U.K., in September 2007. He became a member of academic staff with the State Key Lab of CAD and Computer Graphics, the leading computer graphics lab in China. His recent work has also developed new areas in visual analytics, pattern recognition, image-based rendering, and figure animation. His current research interests include computer graphics, medical visualization, and image processing.

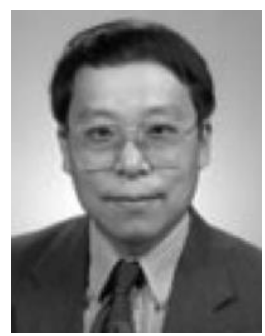

Lida Xu (M'86-SM'11) received the M.S. degree in information science and engineering from the University of Science and Technology of China, Hefei, China, in 1981, and the Ph.D. degree in systems science and engineering from Portland State University, Portland, OR, USA, in 1986. He serves as the Founding Chair of IFIP TC8 WG8.9 and the Founding Chair of the IEEE SMC Society Technical Committee on Enterprise Information Systems.

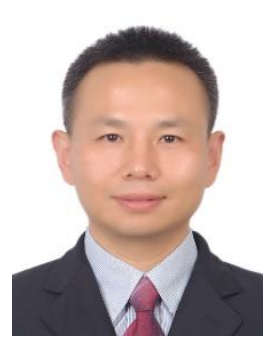

Geyong Min is a Professor of High Performance Computing and Networking in the Department of Mathematics and Computer Science within the College of Engineering, Mathematics and Physical Sciences at the University of Exeter, United Kingdom. He received the $\mathrm{PhD}$ degree in Computing Science from the University of Glasgow, United Kingdom, in 2003, and the B.Sc. degree in Computer Science from Huazhong University of Science and Technology, China, in 1995. His research interests include Future Internet, Computer Networks, Wireless Communications, Multimedia Systems, Information Security, High Performance Computing, Ubiquitous Computing, Modelling and Performance Engineering. 